\title{
Anesthetic Management of a Patient With Ehlers-Danlos Syndrome
}

\author{
Naohiro Ohshita, DDS, PhD,* Masahiko Kanazumi, DDS, PhD,* Kaname Tsuji, DDS, \\ PhD, $\uparrow$ Hiroaki Yoshida, DDS, PhD, $\uparrow$ Shosuke Morita, DDS, PhD, $\uparrow$ Yoshihiro Momota, \\ DDS, PhD,* and Yasuo M. Tsutsumi, MD, PhD $\ddagger$
}

*Department of Anesthesiology, Osaka Dental University, Osaka, Japan, FFirst Department of Oral and Maxillofacial Surgery, Osaka Dental University, Osaka, Japan, and $\neq$ Department of Anesthesiology, Tokushima University, Tokushima, Japan

We describe the case of a 37-year-old woman who had been diagnosed with EhlersDanlos syndrome (EDS) 4 years earlier and was scheduled to undergo removal of synovial chondromatosis in the temporomandibular joint. EDS is a heritable connective tissue disorder and has 6 types. In this case, the patient was classified into EDS hypermobility type. The major clinical feature of this type is joint hypermobility. The patient had sprain or subluxation of the elbows and ankles and dislocation of the knees. Anticipated problems during general anesthesia would be affected by the disease type. For this patient, extra attention was directed to positional injury-induced neuropathy and articular luxation, cutaneous injuries, injuries related to intubation and ventilation, and postoperative pain. Anesthesia was induced with propofol, remifentanil, and rocuronium and maintained with oxygenair-desflurane, propofol, remifentanil, fentanyl, and rocuronium. In this case, the patient was safely managed without adverse events.

Key Words: Ehlers-Danlos syndrome; Hypermobility type; Joint hypermobility and dislocation.

E hlers-Danlos syndrome (EDS) is a heritable connective tissue disorder classified into 6 types: classic, hypermobility, vascular, kyphoscoliosis, arthrochalasis, and dermatosparaxis. ${ }^{1}$ This case is classified into EDS hypermobility type (EDS-HT), and the chief manifestation was joint hypermobility and dislocation. In the branch of dentistry, temporomandibular joint (TMJ) disorder may be one of the complaints. ${ }^{2}$ We report the case of a patient with EDS-HT who underwent removal of synovial chondromatosis in the TMJ under general anesthesia.

\section{CASE REPORT}

A 37-year-old woman (173 cm [68 in], $100 \mathrm{~kg}$, body mass index $=33$ ) with EDS-HT was scheduled for

Received January 14, 2016; accepted for publication April 28, 2016.

Address correspondence to Dr Naohiro Ohshita, Department of Anesthesiology, Osaka Dental University, 1-5-17, Ohtemae, Chuo, Osaka, 540-0008, Japan; oshita@cc.osaka-dent.ac.jp.

Anesth Prog 63:204-207 2016

(C) 2016 by the American Dental Society of Anesthesiology removal of synovial chondromatosis in the TMJ space under general anesthesia.

The patient was a native-born American and had been living in Japan for about 20 years. Her thumb showed joint hypermobility, and her elbow showed hyperextension. Her thumb in conjunction with flexion and extension of her wrist could touch her forearm (Figure A and B). Her skin was not hyperextensible but smooth and velvety. She had suffered sprain or subluxation of the elbows and ankles many times. She had also undergone operations for dislocation of the knees. At present, she had pain into the TMJ, both knees, thumb of the hand, and the iliotibial band. Her medical history included gastroesophageal reflux without current medication use. Thinning hair was being treated with cepharanthine, carpronium chloride hydrate, and deprodone propionate. Electrocardiography revealed sinus arrhythmia with irregular R-R interval and inverted $\mathrm{T}$ waves in leads III and V1. Screening echocardiography revealed normal systolic and diastolic function. 

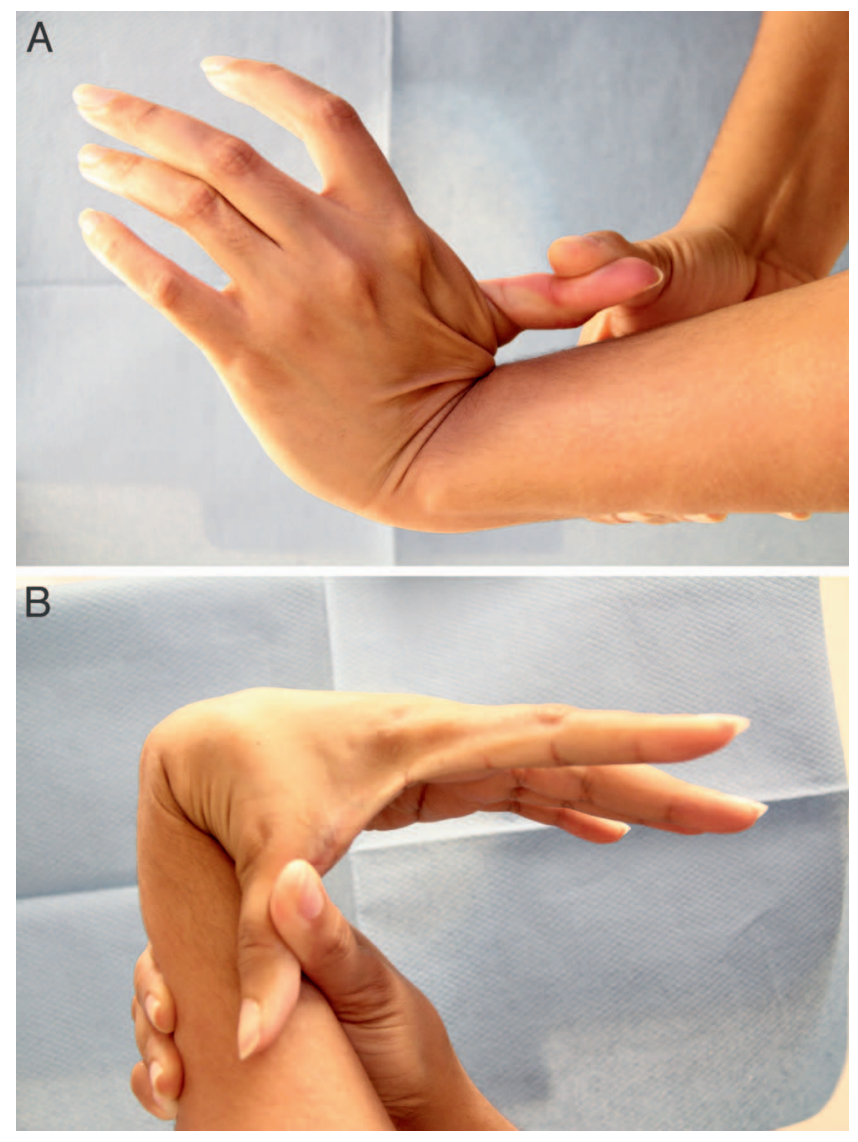

Apposition of the thumbs to the flexor aspect of the forearm.

On the day of the operation, the patient entered the operating room after $500 \mathrm{~mL}$ of acetate Ringer's solution had been infused, and the patient's vital signs were as follows: blood pressure, 115/83 mm Hg; heart rate, 68 beats per minute $(\mathrm{bpm})$ with normal sinus rhythm; and $\mathrm{SpO}_{2}, 99 \%$ in room air. Anesthesia was induced with $180 \mathrm{mg}$ propofol, $0.3 \mu \mathrm{g} / \mathrm{kg} / \mathrm{min}$ remifentanil and $40 \mathrm{mg}$ rocuronium, followed by nasal intubation. Anesthesia was maintained with $6 \%$ desflurane and $0.1 \mu \mathrm{g} / \mathrm{kg} / \mathrm{min}$ remifentanil. For the final 2 hours of the operation, $1 \mathrm{mg} / \mathrm{kg} / \mathrm{h}(16.7 \mu \mathrm{g} / \mathrm{kg} / \mathrm{min})$ propofol was administered to reduce emergence excitement. In addition, we administered $75 \mu \mathrm{g}$ fentanyl and $50 \mathrm{mg}$ flurbiprofen for postoperative analgesia as well as $1.25 \mathrm{mg}$ droperidol and $10 \mathrm{mg}$ metoclopramide to prevent postoperative nausea and vomiting. During the operation, vital signs were generally stable, with a systolic blood pressure of $85-110 \mathrm{~mm} \mathrm{Hg}$, heart rate of $58-110$ bpm without arrhythmia, and $\mathrm{SpO}_{2}$ of 98 $100 \%\left(\mathrm{FiO}_{2}=0.3\right)$. A total of $12 \mathrm{mg}$ ephedrine was administered for transient hypotension. Extubation was uneventful. We completed the anesthesia procedure in 4 hours 35 minutes (operation time 3 hours) with total intraoperative fluid infusion volume of $1100 \mathrm{~mL}$ (1600
$\mathrm{mL}$ total) and total urine volume of $850 \mathrm{~mL}$ with minimal bleeding.

\section{DISCUSSION}

Ehlers-Danlos syndrome was described independently by Ehlers and Danlos in 1901 and 1908, respectively. It was classified into 6 subtypes: classic, hypermobility (EDS-HT), vascular, kyphoscoliosis, arthrochalasis, and dermatosparaxis. ${ }^{1}$ There are also rare variants of EDS with currently unavailable clinical diagnostic criteria (other types of EDS), ${ }^{1}$ but approximately $50 \%$ of patients with EDS have classic type EDS. ${ }^{3}$ In the present case, EDS-HT, also called "EDS type III," mimics joint hypermobility syndrome., ${ }^{4,5}$ EDS-HT and joint hypermobility syndrome are clinically overlapping connective tissue disorders featuring generalized joint hypermobility, muscloskeletal pain, and minor skin features. ${ }^{6}$ EDS-HT and joint hypermobility syndrome may constitute the same clinical entity and likely share the same genetic background, at least in familial cases. Investigations into their relationship are still ongoing. ${ }^{2,4}$ In classic EDS, the causative genes are COL5A1 and A2,COL1A1 and A2, COL3A1, PLOD1, and ADAMTS2, while the causative gene of EDS-HT has not been mapped so far. ${ }^{2}$

In EDS-HT, the hereditary form is thought to be autosomal dominant, and the female:male ratio is about 2:1. ${ }^{4}$ The diagnostic criteria for EDS-HT have major and minor criteria. The major criteria include (1) hyperextensibility and/or smooth, velvety skin and (2) generalized joint hypermobility. Minor criteria are recurring joint dislocation, chronic joint/limb pain, and a positive family history. ${ }^{1}$ Both major criteria, irrespective of the presence/absence of minor criteria, are required for the diagnosis of EDS-HT. ${ }^{4}$ In the present case, the patient was not diagnosed with EDS in childhood but had all the symptoms of EDS: her skin was smooth and velvety, and her thumb showed joint hypermobility. In addition, she had sprain or subluxation of the elbows and ankles countless times and had sustained dislocation of the knees, which had to be treated surgically, several times. Even though her father had Dupuytren's contracture, a chronic, progressive, fibroproliferative disorder of the palm with a predilection for men, ${ }^{7}$ and her older brother was also suspected of having EDS, they had only mild symptoms of ankle sprains.

Our patient currently had chronic pain of the knees and hand, one of the minor criteria for EDS-HT. ${ }^{2,5,8}$ Pain and fatigue are considered common disabling features of EDS-HT, and pain severity is correlated with hypermobility, dislocations, and previous surgery. ${ }^{2}$ In addition, headache, neck pain, and TMJ pain are present in about $30-50 \%$ of patients. ${ }^{2}$ Our patient had 
migraine and TMJ pain caused by synovial chondromatosis, a rare benign condition characterized by the formation of metaplastic cartilaginous nodules in the synovial membrane and TMJ space. ${ }^{9}$ This condition would be nonspecific to EDS.

The frequency of fatigue is influenced by age, with a rate of $28 \%$ in 25-year-olds and $90 \%$ at the age of 40 years. ${ }^{2}$ She also displayed signs of fatiguing easily. In addition, complications in the general anesthetic treatment for EDS vary by each subtype of EDS.

The most risky subtype is EDS vascular type, and EDS vascular type accounts for less than $4 \%$ of all EDS. The vascular type of EDS has the potential for severe morbidity and mortality owing to these patients' propensity to vascular and visceral tissue fragility. ${ }^{10}$ Onefourth of individuals with EDS vascular type experience a significant medical problem: stroke, vascular rupture or dissection, and gastrointestinal perforation or organ rupture by age 20 years and more than $80 \%$ by age 40 years. ${ }^{10-12}$ There is no clear recommendation for either general or regional anesthesia for patients with EDS. ${ }^{13}$ In patients with tissue fragility, epidural or spinal anesthesia would induce hematoma formation or bleeding by needle insertion and catheterization. ${ }^{13,14}$ In obstetric operations, regional versus general anesthesia is chosen based on risk-benefit assessment. ${ }^{14}$ There is no agreement on optimal delivery method, vaginal delivery or cesarean delivery, ${ }^{13}$ and the mortality rates in the parturient with EDS vascular type has been estimated at $12-25 \% .^{14}$

Concerning anesthetic agents, recent reports have indicated that volatile and intravenous anesthesia would be safe. The balanced anesthesia we performed using desflurane, remifentanil, fentanyl, and propofol was successful. ${ }^{13}$ We wanted this patient to maintain anesthesia without additional administration of muscle relaxant (rocuronium), so desflurane was chosen over propofol infusion. In addition, some reports indicate that maintenance of anesthesia with propofol impairs early postoperative lung function and pulse oximetry values more than with desflurane and that increasing obesity decreases pulmonary function with 2 hours or more after propofol anesthesia but not after desflurane anesthesia. ${ }^{15}$ We did, however, use a low-dose propofol infusion to prevent postoperative nausea and vomiting ${ }^{16}$ and to smooth emergence. Adequate prophylaxis of postoperative nausea and vomiting is recommended as spontaneous esophageal rupture has been reported as a result of vomiting in vascular-type EDS. ${ }^{13}$

Clinical features, such as muscle hypoplasia, between inherited connective tissue disorders and congenital myopathies may overlap. In addition, hypotonia and myalgia might be especially common in EDS-HT manifestations. ${ }^{2,17}$ In the present case, our patient had nocturnal muscle cramping as a subjective symptom. These symptoms might be related to the abnormality of the collagen in muscle sheaths rather than the muscle fibers themselves in EDS patients. ${ }^{13}$ As some EDS patients present with muscular weakness, monitoring of neuromuscular blockade would be recommended before emergence of the anesthesia. ${ }^{13}$

We considered that potential problems in patients with EDS-HT included positional injuries, injuries related to intubation and ventilation, and postoperative pain. Concerning positional injuries, excessive mechanical stress, stretching, or pressure increase the vulnerability of peripheral nerves to neuropathy and articular and cutaneous injuries. ${ }^{2}$ We managed this case by using a low rebounding mattress to reduce pressure.

In addition, we attempted to prevent potential postoperative complications such as neck pain and TMJ problems. In this patient, congenital neck flexibility and TMJ luxation were not observed; however, difficult airway management can be affected by subclinical TMJ and neck disorders: accidental joint dislocation during intubation or reduced joint mobility, subclinical spondylosis, and occipital atlanto-axial instability would be issues to be considered in EDS. ${ }^{13}$ We avoided excessive neck extension and excessive mouth opening during intubation. Concerning care related to ventilation, higher inspiratory positive pressures during mechanical ventilation might induce pneumothorax, particularly in patients with tissue fragility. ${ }^{13}$ We used pressure-controlled mode ventilation in this case. Concerning pain relief, TMJ operation would be minimally invasive under general anesthesia, and $<1 \mu \mathrm{g} / \mathrm{kg}$ fentanyl plus $50 \mathrm{mg}$ flurbiprofen were adequate for postoperative analgesia in this case.

In conclusion, complications in general anesthetics for EDS vary with the subtype of EDS. We safely managed this patient with EDS-HT with a balanced anesthesia technique, special care in positioning, and attention to avoiding postoperative nausea and vomiting.

\section{REFERENCES}

1. Beighton P, De Paepe A, Steinmann B, Tsipouras P, Wenstrup RJ. Ehlers-Danlos syndromes: revised nosology, Villefranche, 1997. Ehlers-Danlos National Foundation (USA) and Ehlers-Danlos Support Group (UK). Am J Med Genet. 1998;77:31-37.

2. Castori M, Voermans NC. Neurological manifestations of Ehlers-Danlos syndrome(s): a review. Iran J Neurol. 2014; 13:190-208.

3. Malfait F, De Paepe A. Molecular genetics in classic Ehlers-Danlos syndrome. Am J Med Genet C Semin Med Genet. 2005;139C:17-23. 
4. Castori M, Dordoni C, Valiante M, et al. Nosology and inheritance pattern(s) of joint hypermobility syndrome and Ehlers-Danlos syndrome, hypermobility type: a study of intrafamilial and interfamilial variability in 23 Italian pedigrees. Am J Med Genet A. 2014;164A:3010-3020.

5. Malfait F, Wenstrup RJ, De Paepe A. Clinical and genetic aspects of Ehlers-Danlos syndrome, classic type. Genet Med. 2010;12:597-605.

6. Ghibellini G, Brancati F, Castori M. Neurodevelopmental attributes of joint hypermobility syndrome/Ehlers-Danlos syndrome, hypermobility type: update and perspectives. Am J Med Genet C Semin Med Genet. 2015;169:107-116.

7. Forget NJ, Jerosch-Herold C, Shepstone L, Higgins J. Psychometric evaluation of the Disabilities of the Arm, Shoulder and Hand (DASH) with Dupuytren's contracture: validity evidence using Rasch modeling. BMC Musculoskelet Disord. 2014;15:361.

8. Voermans NC, Knoop H, Bleijenberg G, van Engelen BG. Pain in Ehlers-Danlos syndrome is common, severe, and associated with functional impairment. $J$ Pain Symptom Manage. 2010;40:370-378.

9. Yoshida H, Tsuji K, Oshiro N, Wato M, Morita S. Preliminary report of Ki-67 reactivity in synovial chondromatosis of the temporomandibular joint: an immunohistochemical study. J Craniomaxillofac Surg. 2013;41:473-475.

10. Abayazeed A, Hayman E, Moghadamfalahi M, Cain D. Vascular type Ehlers-Danlos syndrome with fatal spontaneous rupture of a right common iliac artery dissection: case report and review of literature. $J$ Radiol Case Rep. 2014;8:63-69.

11. Callewaert B, Malfait F, Loeys B, De Paepe A. EhlersDanlos syndromes and Marfan syndrome. Best Pract Res Clin Rheumatol. 2008;22:165-189.

12. Dolan P, Sisko F, Riley E. Anesthetic considerations for Ehlers-Danlos syndrome. Anesthesiology. 1980;52:266-269.

13. Wiesmann T, Castori M, Malfait F, Wulf H. Recommendations for anesthesia and perioperative management in patients with Ehlers-Danlos syndrome(s). Orphanet J Rare Dis. 2014;9:109.

14. Fedoruk K, Chong K, Sermer M, Carvalho JC. Anesthetic management of a parturient with hypermobility phenotype but possible vascular genotype Ehlers-Danlos syndrome. Can J Anaesth. 2015;62:1308-1312.

15. Zoremba M, Dette F, Hunecke T, Eberhart L, Braunecker S, Wulf $\mathrm{H}$. A comparison of desflurane versus propofol: the effects on early postoperative lung function in overweight patients. Anesth Analg. 2011;113:63-69.

16. Kawano H, Ohshita N, Katome K, et al. Effects of a novel method of anesthesia combining propofol and volatile anesthesia on the incidence of postoperative nausea and vomiting in patients undergoing laparoscopic gynecological surgery. Braz $J$ Anesthesiol. 2016;66:12-18.

17. Voermans NC, Bonnemann CG, Hamel BC, Jungbluth $\mathrm{H}$, van Engelen BG. Joint hypermobility as a distinctive feature in the differential diagnosis of myopathies. $J$ Neurol. 2009; 256:13-27. 